\title{
Taxol stabilizes gap junctions and reduces ischemic ventricular arrhythmias in rats in vivo
}

\author{
DEGUO WANG ${ }^{1}$, WEN XING $^{1}$, XIN WANG $^{1}$ and HONGJUN ZHU ${ }^{2}$ \\ ${ }^{1}$ Department of Gerontology, Yijishan Hospital of Wannan Medical College, Wuhu, Anhui 241001; \\ ${ }^{2}$ Department of Cardiology, Anhui Provincial Hospital, Hefei, Anhui 231000, P.R. China
}

Received February 10, 2014; Accepted November 12, 2014

DOI: $10.3892 / \mathrm{mmr} .2014 .3137$

\begin{abstract}
The dynamic movements of connexin 43 (Cx43) are regulated by microtubules and their associated proteins. Dysfunction of $\mathrm{Cx} 43$ in the ischemic myocardium is correlated with ventricular arrhythmias (VAs). The present study aimed to determine the effects of microtubules on $\mathrm{Cx} 43$ expression and distribution in myocardial tissue, as well as to examine the susceptibility of the heart to VAs during acute myocardial ischemia and reperfusion. Rats were subject to left coronary artery occlusion for $20 \mathrm{~min}$ followed by $20 \mathrm{~min}$ reperfusion and received taxol at different concentrations $(0.1,0.3$ and $0.9 \mu \mathrm{mol} \cdot \mathrm{kg}^{-1}$ in $0.5 \mathrm{ml}$ saline) intraperitoneally. Monophasic action potentials at the epicardium were recorded and analyzed using an electrocardiogram. Immunoblots and immunofluorescence staining were used to detect tubulin polymerization and $\mathrm{Cx} 43$ expression and distribution. Taxol pretreatment significantly ameliorated the depolymerization of microtubules, improved $\mathrm{Cx} 43$ expression and redistribution, reduced the occurrence of VAs, ameliorated shortening of 90\% repolarization action potential durations (APD) ${ }_{90}$ and improved APD dispersion during myocardial ischemia-reperfusion. The present study demonstrated that taxol reduced ischemic VAs and its mechanism may be correlated with the preservation of Cx43 by stabilizing microtubules.
\end{abstract}

\section{Introduction}

Ventricular arrhythmias (VAs) account for the majority of cardiovascular-associated mortalities in patients with ischemic heart disease. The mechanism of VAs is associated with gap junction (GJ) remodeling involving connexin $43(\mathrm{Cx} 43)$ reduction at the intercalated disc (ID) and $\mathrm{Cx} 43$ lateralization (1). Previous studies have reported that the dynamic movements

Correspondence to: Dr Deguo Wang, Department of Gerontology, Yijishan Hospital of Wannan Medical College, 92 Zheshan Western Road, Wuhu, Anhui 241001, P.R. China

E-mail: wangdeguo@medmail.com.cn

Key words: microtubule, gap junction, taxol, acute myocardial infarction, monophasic action potentials of $\mathrm{Cx} 43$ are associated with microtubules and their associated proteins $(2,3)$. Considering that microtubular disruption is an early cellular reaction to hypoxia in cardiac myocytes $(4,5)$, it is presumed that preservation of the microtubules may affect GJ functions and VAs during myocardial ischemia.

Taxol is a microtubular stabilizer that maintains polymerized microtubular structure and ameliorates hypoxia-induced myocyte injury (6). Taxol-proliferated microtubules are resistant to cold, $\mathrm{Ca}^{2+}$ and colchicines (7). Previous studies have revealed that microtubule stabilizers preserve cardiac function and prevent VAs in an isolated heart $(8,9)$. However, the electrophysiological consequences of in vivo microtubule preservation during myocardial ischemia remains to be elucidated. In the present study, an attempt was made to determine the electrophysiological effects of taxol using an in vivo rat model with myocardial ischemia-reperfusion (IR).

\section{Materials and methods}

Animal model and experimental protocols. Experiments were approved by the Institutional Animal Care and Use Committee of Wannan Medical College (Wuhu, China). A total of 50 Sprague-Dawley rats (250 to $300 \mathrm{~g}$ ) were randomly assigned to five groups: Control group, IR group and three taxol pretreatment groups. In the control and pretreatment groups, normal saline and taxol (Sigma-Aldrich, St. Louis, MO, USA; 0.1, 0.3

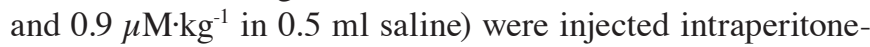
ally (IP) 30 min prior to ischemia induction.

Each rat was anesthetized (pentobarbital sodium, $50 \mathrm{mg} / \mathrm{kg}$ IP; Sigma-Aldrich) and artificially ventilated. Once the pericardium was opened, a 6.0 silk atraumatic suture (Jinghua Co., Shanghai, China) was passed around the left anterior descending coronary artery at the level of the left atrial appendage. Ischemia was induced for $20 \mathrm{~min}$ by ligating the suture, following which it was confirmed by an elevated ST segment in the electrocardiogram (ECG) and changes in myocardial color from red to pale. Reperfusion was initiated by reopening the ligation for $20 \mathrm{~min}$. The control group was established by following the same procedure, but without closing the suture.

ECG and monophasic action potentials (MAPs). ECG traces were recorded continuously, and signals $<10 \mathrm{~Hz}$ and $>100 \mathrm{~Hz}$ were filtered out. Epicardial MAPs were recorded at specific time points intermittently in normal sinus rhythm (10). MAP 
signals were amplified and recorded on a computer for subsequent analysis. A commercially available physiological signals recording and analysis system (RM6240; Chengdu Instrument Company, Chengdu, China) was used to digitalize, store and analyze MAP signals. The software was used to analyze action potential durations $\left(\mathrm{APD}_{90}\right)$ at $90 \%$ of repolarization. Dispersion of action potential duration (APDd) represented the absolute value of the time difference between the maximum and minimum APD.

Analysis of VAs. VAs were analyzed according to Lambeth conventions (11). The score system (9) used to quantify arrhythmias was as follows: $0,<10$ ventricular premature contractions (VPCs); $1, \geq 10$ VPCs; 2, 1-5 episodes of ventricular tachycardia (VT); $3,>5$ episodes of VT or 1 episode of ventricular fibrillation (VF); 4, 2-5 episodes of VF; 5, $>5$ episodes of VF; 6 , VT or VF, or the two together with total combined duration $\leq 300 \mathrm{sec} ; 7, \mathrm{VT}$ or VF, or the two together with total combined duration $>300 \mathrm{sec}$.

Tubulin polymerization assay. A tubulin polymerization assay was performed using a previously described method (6). Myocardial tissue from the ischemic area $(1 \mathrm{~g})$ was minced and homogenized in different buffers. For the total tubulin fraction, the myocardium was homogenized in 1\% SDS buffer containing $10 \mathrm{mM}$ Tris $\cdot \mathrm{HCl}(\mathrm{pH} 7.4), 0.5 \mathrm{mM}$ dithiothreitol and $1 \mathrm{mM} \mathrm{Na}_{3} \mathrm{VO}_{4}$. The mixture was boiled for $5 \mathrm{~min}$ and centrifuged at $16,000 \mathrm{xg}$ at $4^{\circ} \mathrm{C}$ for $10 \mathrm{~min}$ and then the supernatant was saved as the total protein fraction. For the free tubulin heterodimer and polymerized tubulin (microtubule) fractions, the myocardium was homogenized in a microtubule stabilization buffer containing 50\% glycerol, $5 \%$ dimethyl sulfoxide, $10 \mathrm{mM} \mathrm{Na}{ }_{2} \mathrm{HPO}_{4}, 0.5 \mathrm{mM}$ ethylene glycol tetraacetic acid and $0.5 \mathrm{mM} \mathrm{MgSO}_{4}$ and then centrifuged at $100,000 \mathrm{xg}$ at $25^{\circ} \mathrm{C}$ for $20 \mathrm{~min}$. The supernatant was saved as the tubulin heterodimer fraction, whereas the pellet was saved as the tubulin microtubule fraction. For immunoblotting, rabbit polyconal $\alpha$-tubulin $(1: 2,000$; ab125267; Abcam, Cambridge, MA, USA) antibody was used to detect the free tubulin heterodimer and polymerized tubulin.

Western blot analysis. Protein $(100 \mu \mathrm{g})$ was denatured by heating at $95^{\circ} \mathrm{C}$ for $5 \mathrm{~min}$ prior to resolution by SDS-PAGE and transferring to a polyvinylidene difluoride membrane (Shanghai Threebio Technology Co.,Ltd, Shanghai, China). The membrane was blocked in phosphate-buffered saline (Shanghai Threebio Technology Co., Ltd) containing 0.2\% Tween-20 (Shanghai Threebio Technology Co., Ltd) and $5 \%$ skimmed milk for $2 \mathrm{~h}$ at $37^{\circ} \mathrm{C}$ and incubated overnight at $4^{\circ} \mathrm{C}$ with primary polyclonal antibodies (rabbit anti-Cx43; 1:2,000; ab11370; Abcam). The housekeeping protein, glyceraldehyde-3-phosphate dehydrogenase (rabbit anti-GAPDH polyclonal antibody; ab9485; Abcam) was used as a loading control. Antibody binding was detected using horseradish peroxidase-conjugated secondary antibody $(1: 2,000$; Sigma-Aldrich) and visualized using an enhanced chemiluminescence kit (Chemicon, Temecula, CA, USA).

Immunofluorescence staining. Frozen tissue sections fixed in cold acetone $\left(-20^{\circ} \mathrm{C}\right.$ for $\left.5 \mathrm{~min}\right)$ were used for immuno- fluorescence staining. Nonspecific antibody binding sites were blocked with $5 \%$ fetal bovine serum for $60 \mathrm{~min}$. Tissue sections were labeled for $16 \mathrm{~h}$ at $4^{\circ} \mathrm{C}$ with rabbit polyclonal anti-Cx43 (Cx43; 1:200, Abcam). Anti-rabbit immunoglobulin G conjugated with fluorescein isothiocyanate was used for detection and images were observed using a Zeiss confocal microscope (LSM-510; Carl Zeiss, Gottingen, Germany).

Statistical analysis. Statistical analysis was performed using SPSS 16.0 software (SPSS, Inc., Chicago, IL, USA). Data are expressed as the mean \pm standard error of the mean and were analyzed using analysis of variance followed by Newman-Keul's t-test. $\mathrm{P}<0.05$ was considered to indicate a statistically significant difference.

\section{Results}

Effects of taxol on IR-induced VAs. In a preliminary experiment, it was observed that $>2 \mu \mathrm{M}$ taxol may lead to lethal ventricular bradycardias. Thus, the doses of taxol were reduced to $0.1,0.3$ and $0.9 \mu \mathrm{M}$. In the control group, $6 / 10$ rats had $\geq 10$ VPCs and only 1 had an episode of VT. In the IR group, all animals had $>5$ episodes of VT or several episodes of VF. The spontaneous VTs/VFs appeared most frequently ( 5 to $10 \mathrm{~min}$ ) after ligation and then recovered gradually. Within 2 min after reperfusion, VPCs/VTs/VFs occurred transiently and automatically recovered rapidly. In three taxol treatment groups, fewer animals had VTs and VFs than in the IR group. However, no difference in VAs during reperfusion periods between the IR group and taxol-treated groups was observed (Fig. 1; Table I). The severity of VAs was significantly decreased in taxol-treated groups in a dose-dependent manner compared with the IR group based on the VA score system $(\mathrm{P}<0.01$; Fig. 1$)$.

Effects of taxol on IR-induced MAP changes. The MAP duration at $\mathrm{APD}_{90}$ of the ischemic epicardium in the IR group was significantly shorter than that of the control group (Fig. 2). When perfusion was restored, the $\mathrm{APD}_{90}$ rapidly recovered within 5 min and returned to baseline levels. The APDd was significantly increased in the IR group compared with that in the control group. Taxol treatment partially but significantly restored $\mathrm{APD}_{90}$ and reduced APDd during ischemia in the pretreatment groups. However, no significant difference in $\mathrm{APD}_{90}$ was observed during the reperfusion periods between the IR group and taxol-treated groups.

Effects of taxol on IR-induced microtubular depolymerization. The relative levels of free tubulin in the IR group increased significantly after the heart was exposed to IR compared with the control group (Fig. 3). In addition, polymerized tubulin in the IR group significantly decreased. Taxol treatment maintained tubulin polymerization significantly and reduced free tubulin in a dose-dependent manner $(\mathrm{P}<0.01)$.

Effects of taxol on IR-induced Cx43 distribution in the heart. The $\mathrm{Cx} 43$ staining signal in the non-ischemic heart was concentrated at the IDs (red arrows, Fig. 4A), whereas little $\mathrm{Cx} 43$ was located in the lateral cardiomyocyte surfaces (yellow arrows, Fig. 4A). In the hearts of the IR group, the 
Table I. Effects of taxol on ischemia/reperfusion-induced ventricular arrhythmias.

\begin{tabular}{|c|c|c|c|c|c|c|c|}
\hline \multirow[b]{2}{*}{ Group } & \multicolumn{3}{|c|}{ Ischemia } & \multicolumn{3}{|c|}{ Reperfusion } & \multirow[b]{2}{*}{ VA Score } \\
\hline & VTs & VFs & VPCs & VTs & VFs & VPCs & \\
\hline Control & $1 / 10$ & 0 & $7 / 10$ & - & - & - & $0.8 \pm 0.63$ \\
\hline IR & $10 / 10^{\mathrm{a}}$ & $6 / 10^{\mathrm{a}}$ & $10 / 10$ & $8 / 10$ & $3 / 10$ & $10 / 10$ & $3.3 \pm 0.95^{\mathrm{a}}$ \\
\hline $\mathrm{IR}+0.1 \mu \mathrm{M}$ taxol & $10 / 10^{\mathrm{a}}$ & $4 / 10^{\mathrm{a}}$ & $10 / 10$ & $7 / 10$ & $3 / 10$ & $10 / 10$ & $2.7 \pm 0.48^{\mathrm{ab}}$ \\
\hline $\mathrm{IR}+0.3 \mu \mathrm{M}$ taxol & $10 / 10^{\mathrm{a}}$ & $3 / 10^{\mathrm{ab}}$ & $10 / 10$ & $6 / 10$ & $2 / 10$ & $10 / 10$ & $2.5 \pm 0.53^{\mathrm{ab}}$ \\
\hline $\mathrm{IR}+0.9 \mu \mathrm{M}$ taxol & $7 / 10^{\mathrm{a}}$ & $0 / 10^{\mathrm{ab}}$ & $10 / 10$ & $6 / 10$ & $2 / 10$ & $10 / 10$ & $1.7 \pm 0.45^{\mathrm{ab}}$ \\
\hline
\end{tabular}

${ }^{a} \mathrm{P}<0.05$ vs. control group and ${ }^{\mathrm{b}} \mathrm{P}<0.05$ vs. IR group. VT, ventricular tachycardia; VF, ventricular fibrillation; VPC, ventricular premature contractions; IR, ischemia-reperfusion.
L ead II

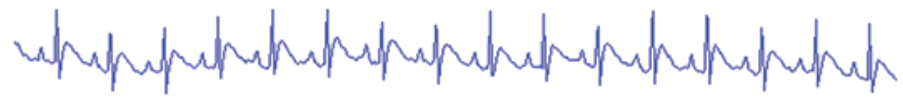
Pre-ischemia

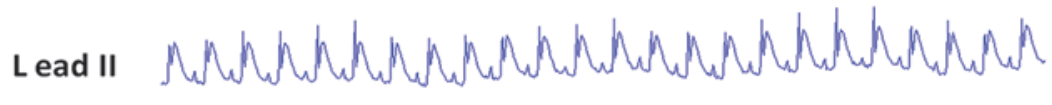
Ischemia-
reperfusion
L ead II

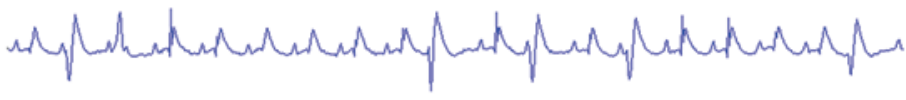
Ventricular
bigeminy
L ead II

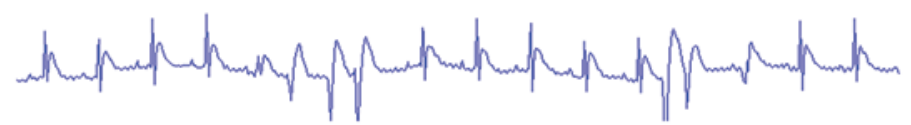
Ventricular
couplet

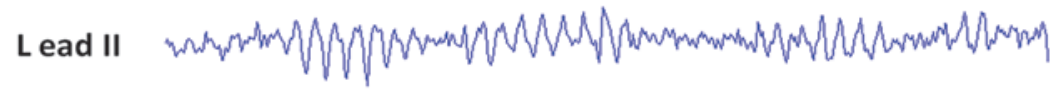
Ventricular
fibrillation

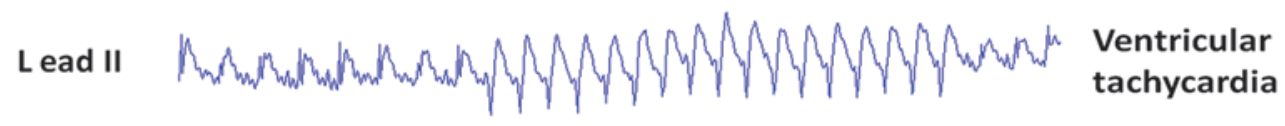

Figure 1. Ventricular arrhythmias induced by coronary occlusion and reperfusion. During left anterior descending coronary occlusion and reperfusion, animals developed ventricular arrhythmias, including ventricular bigeminy, ventricular couplet, ventricular tachycardia and ventricular fibrillation.

Cx43 signal was markedly reduced in ID and redistributed to the lateral cardiomyocyte surfaces (Fig. 4B). The hearts subjected to taxol pretreatment exhibited significantly improved Cx43 distribution compared with the IR groups (Fig. 4C, D and E).

Effects of taxol on IR-induced Cx43 expression. The relative levels of $\mathrm{Cx} 43$ protein expression in myocardial tissue were reduced significantly in the IR group compared with the control group (Fig. 5). Taxol treatment significantly improved Cx43 expression in a dose-dependent manner $(\mathrm{P}<0.01)$.

\section{Discussion}

Microtubules are important components of the cytoskeleton. They have important functions in protein synthesis, intracel- lular trafficking and intracellular signaling. During these processes, microtubules require a dynamic balance between free tubulin and polymerized dimers in the cytoplasm $(12,13)$. Previous studies have demonstrated that microtubules are responsible for regulating $\mathrm{Ca}^{2+}$ channels, APD and membrane potential (14). In the present study, it was observed that susceptibility to VAs increased in an in vivo rat model with IR, however the susceptibility was also partly but significantly reduced by taxol pretreatment in a dose-dependent manner. The present results are in agreement with previous ex vivo experiments $(8,9)$, indicating that stabilizing microtubules have significant anti-arrhythmic effects during myocardial IR in vivo.

Previous studies have revealed that during myocardial ischemia, either APD shortening or APDd enlargement generates pro-arrhythmic substrates $(15,16)$. APD duration undergoes a 
A

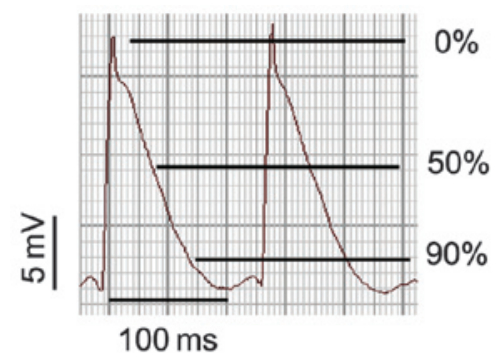

B

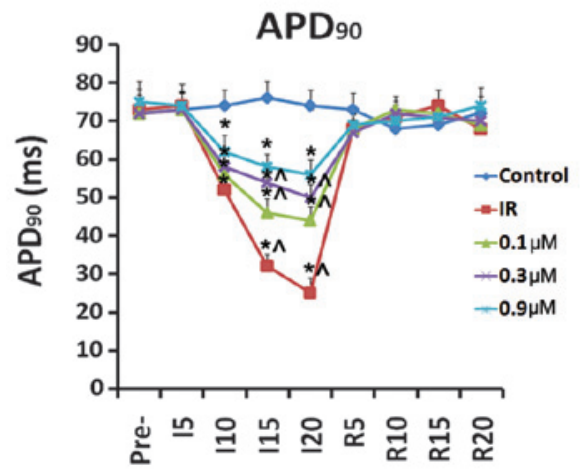

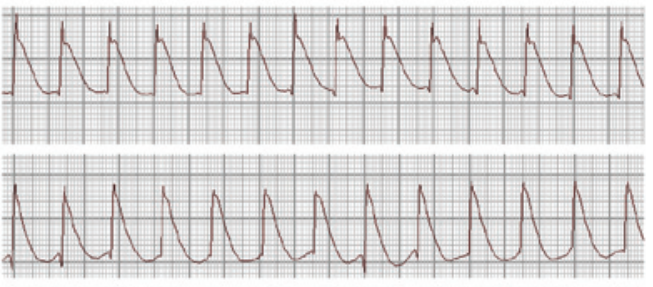

C

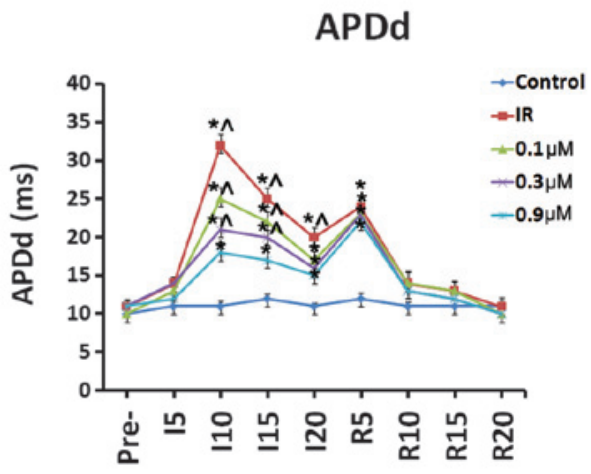

Figure 2. Epicardial monophasic APD and APDd is altered by IR. (A) MAP was recorded from the ischemia-reperfusion zone (apical). (B) $\mathrm{APD}_{90}$ is the $90 \%$ repolarization of MAP durations; (C) APDd is the absolute value of the time difference between the maximum and minimum of APD. "P $<0.05$, versus the control group; ${ }^{\wedge} \mathrm{P}<0.05$, versus the IR group. IR, ischemia-reperfusion; APD, action potential duration; APDd, dispersion of action potential duration; $\mathrm{APD}_{90}$ action potential duration $90 \%$ of repolarization; MAP, monophasic action potentials.

A

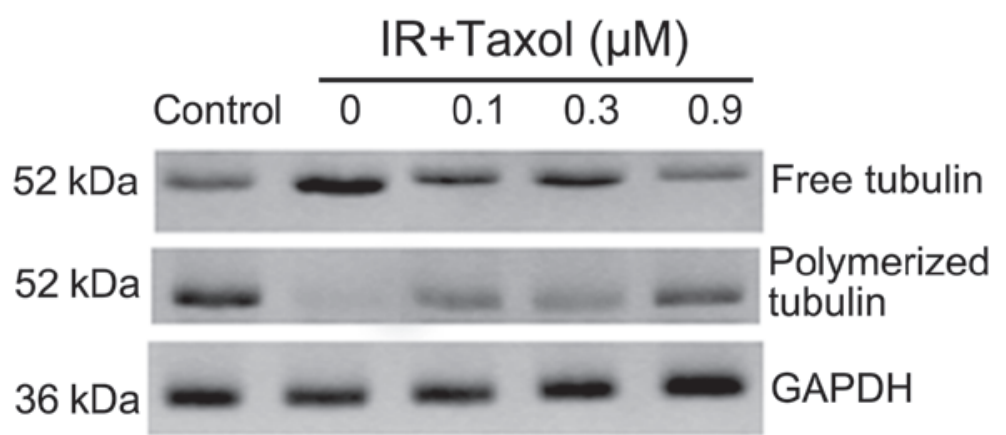

B

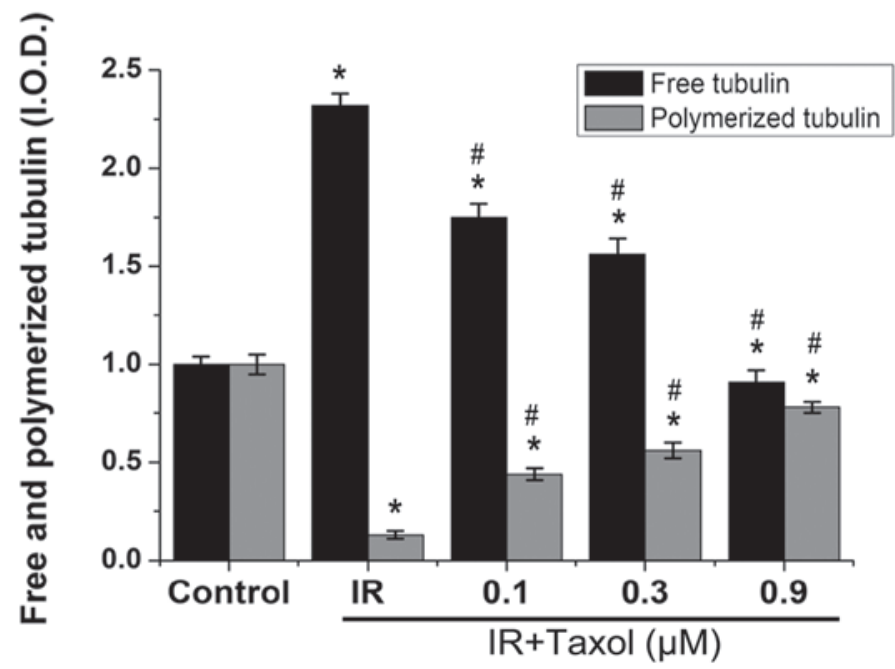

Figure 3. Taxol protects microtubules from depolymerization in the ischemic myocardium. Immunoblot examples from the apex of the left ventricle after myocardial ischemia-reperfusion. (A) Monoclonal anti-GAPDH antibody was used to load the control blot. (B) Relative quantitative levels of free and polymerized tubulin expression (IOD). ${ }^{~} \mathrm{P}<0.05$, versus the control group; ${ }^{\prime} \mathrm{P}<0.01$, versus the IR group. IR, ischemia-reperfusion; IOD, integrated optical density. 
A

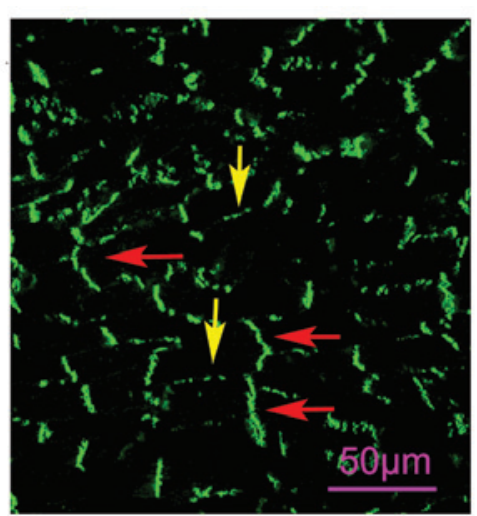

D

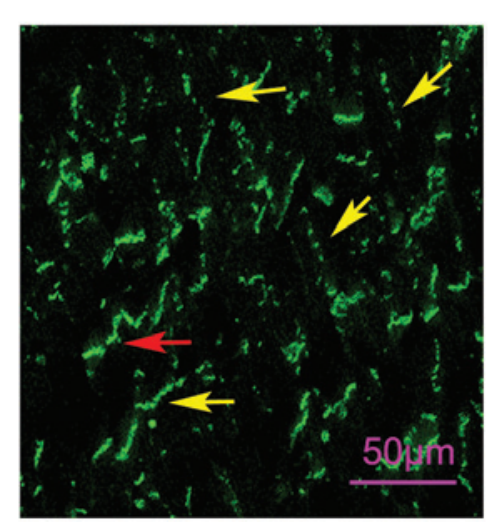

B

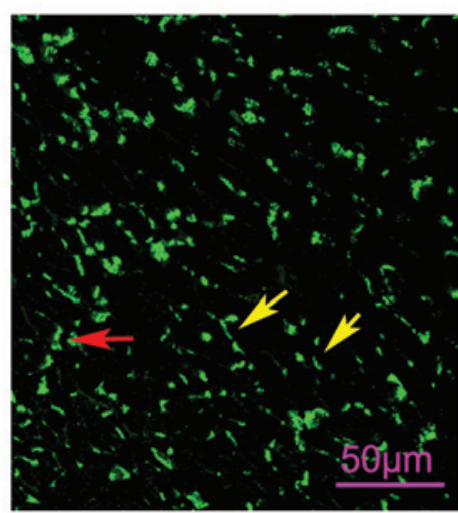

E

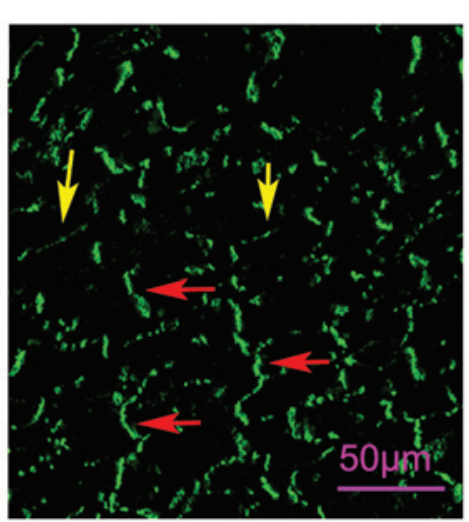

C

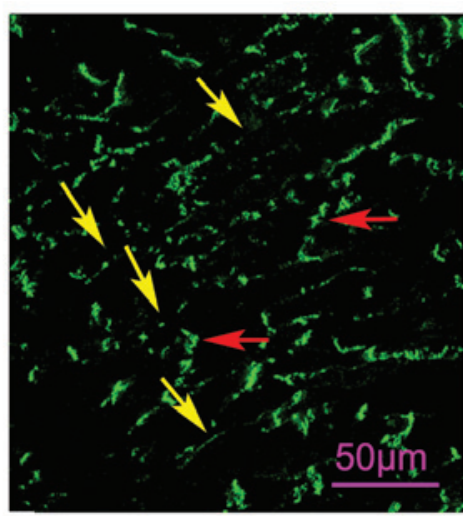

Figure 4. Taxol stabilizes Cx43 distribution in the ischemic myocardium. Representative immunofluorescence Cx43 images of sections from the apex of left ventricles from the (A) control group, (B) IR group and (C, D and E) $0.1,0.3$ and $0.9 \mu \mathrm{M}$ taxol pre-treatment groups, respectively. Red arrows indicate Cx 43 in intercalated disks, whereas yellow arrows indicate lateralized Cx43 in cardiac myocyte surfaces. IR, ischemia-reperfusion; Cx43, connexin 43.

A

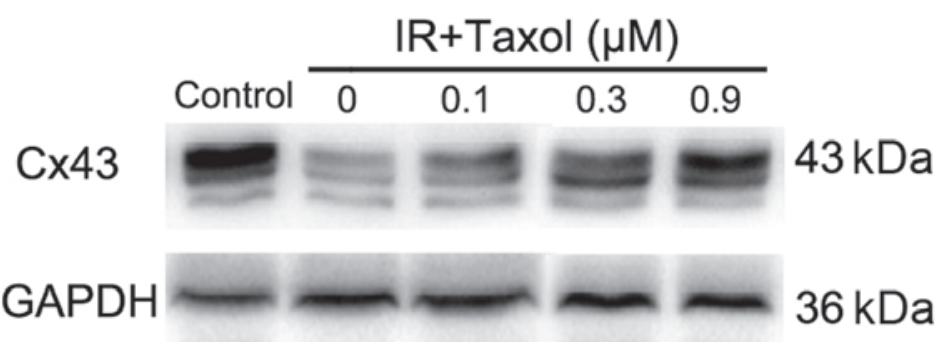

B

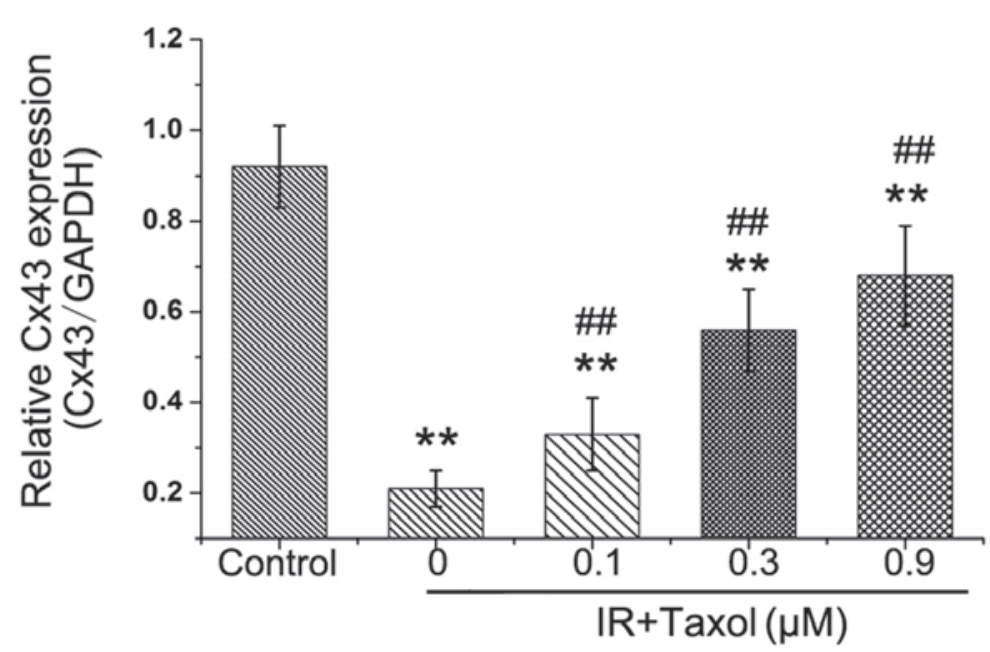

Figure 5. Taxol preserves Cx43 expression in the ischemic myocardium. Representative (A) western blots and (B) relative quantitative levels of Cx43 expression. ${ }^{* *} \mathrm{P}<0.01$, versus the control group; ${ }^{\# \#} \mathrm{P}<0.01$, versus the IR group. IR, ischemia-reperfusion; $\mathrm{Cx} 43$, connexin 43 . 
temporary prolongation and then gradually recovers following reperfusion (17). Considering that microtubules regulate APD and membrane potential (14), taxol may also affect APD duration and APDd during myocardial IR. In the present study, $\mathrm{APD}_{90}$ shortened rapidly following coronary occlusion, however was restored within $5 \mathrm{~min}$ after reperfusion. Taxol treatment significantly ameliorated ischemia-induced APD shortening and APDd, which can reduce ischemia-induced arrhythmias. Thus the beneficial effects of taxol on ischemia-induced arrhythmias may be due to the improvement in APD changes. In cultured myocytes, microtubular depolymerization is an early cellular reaction to hypoxia (4). Acute hypoxia leads to the collapse of microtubular networks and reversible cellular damage (18). The interruption of microtubules occurs prior to mitochondrial damage and myocyte injury $(19,20)$. Taxol may stabilize polymerized microtubules and ameliorate hypoxia-induced myocyte injury (6). The present data demonstrated that taxol treatment significantly preserved polymerized tubulin and reduced free tubulin during IR.

GJ remodeling is associated with cardiac arrhythmias during myocardial ischemia. Previous studies have revealed that oxidative stress can affect microtubules and lead to subsequent perturbation of $\mathrm{Cx} 43$ delivery to the plasma membrane in the human and mouse myocardium $(2,3)$. In the present study, it was observed that in the normal ventricular tissue, $\mathrm{Cx} 43$ was localized at IDs. It was also identified that taxol pretreatment significantly improved Cx43 distribution and expression in the ischemic myocardial tissue. The stabilization of Cx43 during ischemia may be associated with reduction of VAs through taxol pretreatment.

The present study has several limitations. The phosphorylation levels of $\mathrm{Cx} 43$ that may modulate GJ function during ischemia were not detected (21). In addition, the present study was not able to exclude the possibility that GJ preservation of taxol was secondary to the reduction of oxidative stress, which can reduce the levels of connexin passing to the plasma membrane (22). Taxol may also be able to modulate ion channels (14). Therefore, further studies should focus on identifying the ion channels preserved by taxol during ischemic injury.

The present study demonstrated that taxol pretreatment may stabilize microtubules, preserve GJs and reduce the severity of VAs during myocardial IR. These findings indicated that microtubule stabilization may be of benefit in modifying the VA susceptibility of ischemic hearts.

\section{Acknowledgements}

The present study was supported by grants from the National Natural Science Foundation of Anhui Province (grant nos. 1208085QH156 and 1208085MH129) and the National Natural Science Foundation of China (grant no. 81200142).

\section{References}

1. Kieken F, Mutsaers N, Dolmatova E, et al: Structural and molecular mechanisms of gap junction remodeling in epicardial border zone myocytes following myocardial infarction. Circ Res 104: 1103-1112, 2009.
2. Shaw RM, Fay AJ, Puthenveedu MA, von Zastrow M, Jan YN and Jan LY: Microtubule plus-end-tracking proteins target gap junctions directly from the cell interior to adherens junctions. Cell 128: 547-560, 2007.

3. Smyth JW, Hong TT, Gao D, et al: Limited forward trafficking of connexin 43 reduces cell-cell coupling in stressed human and mouse myocardium. J Clin Invest 120: 266-279, 2010.

4. Vandroux D, Schaeffer C, Tissier C, et al: Microtubule alteration is an early cellular reaction to the metabolic challenge in ischemic cardiomyocytes. Mol Cell Biochem 258: 99-108, 2004.

5. Devillard L, Vandroux D, Tissier C, et al: Involvement of microtubules in the tolerance of cardiomyocytes to cold ischemia-reperfusion. Mol Cell Biochem 307: 149-157, 2008.

6. Teng M, Dang YM, Zhang JP, et al: Microtubular stability affects cardiomyocyte glycolysis by HIF-lalpha expression and endonuclear aggregation during early stages of hypoxia. Am J Physiol Heart Circ Physiol 298: H1919-H1931, 2010.

7. Howarth FC, Calaghan SC, Boyett MR and White E: Effect of the microtubule polymerizing agent taxol on contraction, $\mathrm{Ca}^{2+}$ transient and L-type $\mathrm{Ca}^{2+}$ current in rat ventricular myocytes. J Physiol 516 (Pt 2): 409-419, 1999.

8. Xiao J, Zhao H, Liang D, et al: Taxol, a microtubule stabilizer, improves cardiac contractile function during ischemia in vitro. Pharmacology 85: 301-310, 2010.

9. Xiao J, Cao H, Liang D, et al: Taxol, a microtubule stabilizer, prevents ischemic ventricular arrhythmias in rats. J Cell Mol Med 15: 1166-1176, 2011.

10. Franz MR: Current status of monophasic action potential recording: theories, measurements and interpretations. Cardiovasc Res 41: 25-40, 1999.

11. Walker MJ, Curtis MJ, Hearse DJ, et al: The Lambeth Conventions: guidelines for the study of arrhythmias in ischaemia infarction, and reperfusion. Cardiovasc Res 22: 447-455, 1988.

12. Kawakami K, Chiba T, Katagiri N, et al: Paclitaxel increases high voltage-dependent calcium channel current in dorsal root ganglion neurons of the rat. J Pharmacol Sci 120: 187-195, 2012.

13. De Vuyst E, Boengler K, Antoons G, Sipido KR, Schulz R and Leybaert L: Pharmacological modulation of connexin-formed channels in cardiac pathophysiology. Br J Pharmacol 163: 469-483, 2011.

14. Calaghan SC, Le Guennec JY and White E: Cytoskeletal modulation of electrical and mechanical activity in cardiac myocytes. Prog Biophys Mol Biol 84: 29-59, 2004.

15. Tsuburaya R, Yasuda S, Ito Y, et al: Eicosapentaenoic acid reduces ischemic ventricular fibrillation via altering monophasic action potential in pigs. J Mol Cell Cardiol 51: 329-336, 2011.

16. Chen Y, Zhang Q, Liao YH, et al: Effect of tumor necrosis factor-alpha on neutralization of ventricular fibrillation in rats with acute myocardial infarction. Mediators Inflamm 2011: 565238,2011

17. Guo X, Gao X, Wang Y, Peng L, Zhu Y and Wang S: IKs protects from ventricular arrhythmia during cardiac ischemia and reperfusion in rabbits by preserving the repolarization reserve. PLoS One 7: e31545, 2012.

18. Hori M, Sato H, Kitakaze M, et al: Beta-adrenergic stimulation disassembles microtubules in neonatal rat cultured cardiomyocytes through intracellular $\mathrm{Ca}^{2+}$ overload. Circ Res 75: 324-334, 1994.

19. Saetersdal T, Greve G and Dalen H: Associations between beta-tubulin and mitochondria in adult isolated heart myocytes as shown by immunofluorescence and immunoelectron microscopy. Histochemistry 95: 1-10, 1990.

20. Devillard L, Vandroux D, Tissier C, et al: Tubulin ligands suggest a microtubule-NADPH oxidase relationship in postischemic cardiomyocytes. Eur J Pharmacol 548: 64-73, 2006.

21. Beardslee MA, Lerner DL, Tadros PN, et al: Dephosphorylation and intracellular redistribution of ventricular connexin 43 during electrical uncoupling induced by ischemia. Circ Res 87: 656-662, 2000

22. Smyth JW, Hong TT, Gao D, et al: Limited forward trafficking of connexin 43 reduces cell-cell coupling in stressed human and mouse myocardium. J Clin Invest 120: 266-279, 2010. 